\title{
THE STRONG MAXIMUM MODULUS THEOREM FOR ANALYTIC FUNCTIONS INTO A BANACH SPACE ${ }^{1}$
}

\section{EDWARD THORP AND ROBERT WHITLEY}

1. Introduction. Let $D$ be a domain (an open connected subset) in the complex plane and let $f$ be a complex-valued analytic function on $D$. Then the classical maximum modulus theorem says that either $|f(z)|$ has no maximum on $D$ or that $|f(z)|$ is a constant on $D$. If $f(z) \mid$ is a constant, it follows readily that $f(z)$ is itself constant.

If $f$ has values in a complex $B$-space, it is well known [5, p. 230], or $[6$, p. 100] that the theorem holds. However, the strong form of the maximum modulus theorem, where if $|f(z)|$ is constant then $f(z)$ is constant, is no longer true in general. This is illustrated by the following simple example $[6$, p. 100]. Let $D$ be the open unit disc and define $f: D \rightarrow l_{\infty}^{2}$ by $f(z)=(1, z)$. Then $f$ is analytic, not constant, and $|f(z)|=1$ for all $z$ in $D$. (Notation throughout this paper, such as $l_{\infty}^{2}$, follows [5] wherever possible.)

We show below that the strong form of the maximum modulus theorem always holds for a $B$-space $X$ if and only if each point of norm one is a "complex extreme point" of the unit sphere of $X$. In particular, the theorem holds for strictly convex (i.e. rotund) spaces. We also discuss geometrical conditions on the range of $f$ under which the theorem holds for that $f$. Lastly, we discuss the theorem for particular $B$-spaces.

2. Complex extreme points. The notion of complex extreme point is central to our results. To motivate it, recall that an extreme point $e$ of a convex set $K$, hereafter called a real extreme point, is one which cannot be in the interior of a segment in $K$. That is, if $e=a x+(1-a) y$ for $0<a<1$, with $x \neq y$, then either $x$ or $y$ is not in $K$. Equivalently, $e$ is a real extreme point if it is not the midpoint of any segment in $K$. Thus a real "disc" centered at $e$ "sticks out of" $K$, no matter in which direction it is tilted. By analogy we have the following.

Definition 2.1. A point $e$ of a convex subset $K$ of a complex $B$ space $X$ is a complex extreme point of $K$ if $\{e+z y:|z| \leqq 1\} \subset K$ for $y$ in $X$ implies that $y=0$.

REMARKS. This is easily seen to be equivalent to requiring only $|z|=1$, or even to requiring only that $z= \pm 1, \pm i$. The set of complex extreme points always contains the set of real extreme points for a

Presented to the Society, January 27, 1967; received by the editors June 29, 1966.

1 The research for this paper was supported in part by the Air Force Office of Scientific Research, under Grants AFOSR 457-63 and 1113-66. 
convex set $K$. Thus the Kreln-Mil'man theorem holds a fortiori for the set of complex extreme points.

\section{The strong form of the maximum modulus theorem.}

Theorem 3.1. Let $X$ be a complex Banach space such that each point on the surface of the unit sphere is a complex extreme point of the unit sphere. Then the strong form of the maximum modulus theorem holds, i.e. for $D$ a domain and $f: D \rightarrow X$ an analytic function, either $|f(z)|$ has no maximum on $D$ or $f(z)$ is constant on $D$.

Conversely, if the surface of the unit sphere of $X$ contains a point which is not a complex extreme point of the sphere, then there is a nonconstant analytic function $f$ mapping the open unit disc into $X$ yet satisfying $|f(z)|=1$ for all $z$ in the disc.

Proof. For the converse, suppose that $|x|=1$ and $x$ is not a complex extreme point. Choose $y \neq 0$ such that $|x+z y| \leqq 1$ for all $|z| \leqq 1$. If $\left|x+z_{0} y\right|<1$ for some $\left|z_{0}\right| \leqq 1$, we have $|x| \leqq\left(\left|x+z_{0} y\right|+\left|x-z_{0} y\right|\right) / 2$ $<1$, a contradiction. Thus $|x+z y|=1$ for all $|z| \leqq 1$ and the function $f(z)=x+z y,|z|<1$, satisfies the requirements of the converse.

Now assume that every point on the unit sphere of $X$ is a complex extreme point of the unit sphere. Suppose $f$ is an analytic map of a domain $D$ into $X$ and that $|f(z)|$ attains its maximum for some $z$ in $D$. Then, by the usual form of the theorem, $|f(z)|=a$, a constant, for $z$ in $D$. If $a=0$, then $f(z)=0$ and so is constant. If $a \neq 0, f / a$ has norm 1 in $D$ so, to show $f$ is constant, we may suppose that $|f(z)|=1$ for $z$ in $D$. By the identity theorem, it is enough to show that $f$ is constant in some neighborhood in $D$, and by a change of variable, we may suppose that the neighborhood is the interior of the unit disc. So Theorem 3.1 follows directly from Theorem 3.2, below.

THEOREM 3.2. Let $f$ be an analytic function mapping the interior of the unit disc into a complex $B$-space $X$ and suppose that $|f(z)|=1$ for $|z|<1$. If $f(0)$ is a complex extreme point of the unit sphere in $X$, then $f(z)$ is constant for $|z|<1$.

Note that Theorem 3.2 remains true when $f(0)$ is replaced by $f(z)$, an arbitrary point in the range of $f$. This follows readily from a change of variable and the identity theorem.

The proof of Theorem 3.2 depends on the next lemma.

Lemma 3.3. Let $D$ be a domain in the complex plane, $X$ a complex $B$-space, and $f: D \rightarrow X$ an analytic function with $|f(z)|=1$ for all $z$ in $D$. Then for each point $y$ in $\mathrm{cl}(\operatorname{co} f(D))$, the closed convex hull of the range of $f$, we have $|y|=1$. 
Proof. Let $y$ be in $\operatorname{co}(f(D))$. Then there are positive numbers $a_{1}, \cdots, a_{n}$ with $\sum a_{i}=1$ and points $z_{1}, \cdots, z_{n}$ in $D$ such that $y=\sum a_{i} f\left(z_{i}\right)$. By the Hahn-Banach theorem there is a continuous linear functional $x^{*}$ of norm one with $x^{*}\left(f\left(z_{1}\right)\right)=1$. Since $\left|x^{*}(f(z))\right|$ $\leqq\left|x^{*}\right||f(z)|=1$ for all $z$ in $D, x^{*} f$ is an analytic function on $D$ which attains its maximum at $z_{1}$. From the classical maximum modulus theorem $x^{*} f(z)=1$ on $D$. Thus $x^{*}(y)=\sum a_{i} x^{*} f\left(z_{i}\right)=1$ which implies that $|y| \geqq 1$. Since $|y| \leqq \sum a_{i}\left|f\left(z_{i}\right)\right|=1,|y|=1$. It follows at once that $\mathrm{cl}(\operatorname{co} f(D))$ also consists of points of norm 1, which completes the proof.

Recall that a Banach space is strictly convex (also called rotund) if and only if each point on the surface of the unit sphere is a real extreme point. Corollary 3.4 will follow at once when we complete the proof of Theorem 3.1. But we point out now an immediate direct proof.

Corollary 3.4. Let $X$ be a strictly convex complex B-space. The strong form of the maximum modulus theorem holds for analytic functions with values in $X$.

Proof. Use Lemma 3.3 and the fact that a convex set which lies on the surface of the unit sphere in a strictly convex space must consist of one point.

Proof of Theorem 3.2. Let $D=\{z:|z|<1\}$. Write $f(z)=x_{0}+g(z)$ where $g(0)=0$. We have $\mathrm{cl}(\operatorname{co} f(D))=x_{0}+\mathrm{cl}(\operatorname{co} g(D))$ and $\mathrm{cl}(\operatorname{cog} g(D))$ $=\left\{\sum a_{i} g\left(z_{i}\right): z_{i} \in D, a_{i} \geqq 0\right.$ and $\left.\sum a_{i} \leqq 1\right\}$. The condition $\sum a_{i}=1$ can be replaced by $\sum a_{i} \leqq 1$ because 0 is in $g(D)$.

The method of proof is to assume that $f(0)$ is a complex extreme point. We then show that $g$ is zero by supposing that $g$ is not zero and then finding a nonzero element $w$ in $\operatorname{cl}(\operatorname{cog} g(D))$ with the property that $z w$ is in $\operatorname{cl}(\operatorname{cog} g(D))$ for $|z|<r \neq 0$. By Lemma 3.3 it then follows that $\left|x_{0}+z r w\right|=1$ for all $z$ with $|z|<1$, and this contradicts the assumption that $f(0)=x_{0}$ is a complex extreme point of the unit sphere in $X$.

Since $g$ is analytic we can write $g(z)=\sum_{n=1}^{\infty} c_{n} z^{n}$, for $|z|<1$, where the $c_{n}$ are in $X\left[6\right.$, p. 97]. We suppose that $c_{n_{0}}$ is the first nonzero coefficient of $g(z)$. Let $w_{1}, w_{2}, \cdots, w_{n_{0}}$ be the $n_{0}$ th roots of unity. For $|z|<1$ and any determination of $z^{1 / n 0}$ we have

$$
\frac{1}{n_{0}} \sum_{i=1}^{n_{0}} g\left(z^{1 / n 0} w_{i}\right)=c_{n_{0}} z+c_{2 n_{0}} z^{2}+\cdots=g_{0}(z) .
$$

For simplicity we set $g_{0}(z)=\sum_{n=1}^{\infty} b_{n} z^{n}$ for $|z|<1$. The function $g_{0}$ is 
analytic since it has a convergent power series expansion, and $g_{0}(z)$ is in $\operatorname{co}(g(D))$ for $z$ in $D$, so $\mathrm{cl}\left(\operatorname{co~} g_{0}(D)\right) \subset \mathrm{cl}(\operatorname{co~} g(D))$. The proof of Theorem 3.2 will thus be complete if we can show that $b_{1} z$ is in $\operatorname{co} g_{0}(D)$ for $|z|$ sufficiently small. To do this we need the next lemma.

Lemma 3.5. There is a constant $M$ such that for each integer $n$ we can find complex numbers $z_{1}, \cdots, z_{n}$ with

(i) $\left|z_{i}\right|<M$ for $i=1,2, \cdots, n$,

(ii) $(1 / n) \sum_{i=1}^{n} z_{i}=1$,

(iii) $(1 / n) \sum_{i=1}^{n} z_{i}^{p}=0, p=2,3, \cdots, n$.

Proof. For a polynomial $x^{n}+a_{1} x^{n-1}+\cdots+a_{n}$ with roots $r_{1}, \cdots$, $r_{n}$, let $R_{p}=\sum_{j=1}^{n}\left(r_{j}\right)^{p}, p=1,2, \cdots$ Newton's identities are $[\mathbf{8}$, pp. 260-262]:

$$
\begin{aligned}
& R_{1}=-a_{1} \\
& R_{2}+a_{1} R_{1}=-2 a_{2} \\
& \cdots \ldots \ldots \ldots \ldots \ldots \ldots \ldots \ldots \\
& R_{n}+a_{1} R_{n-1}+\cdots+a_{n-1} R_{1}=-n a_{n} .
\end{aligned}
$$

From these identities we see that the polynomial

$$
Q_{n}(x)=x^{n}-n x^{n-1}+\frac{n^{2}}{2 !} x^{n-2}-\cdots+\frac{(-1)^{n} n^{n}}{n !}
$$

has roots $z_{1}, z_{2}, \cdots, z_{n}$ which satisfy (ii) and (iii) above. We write $Q_{n}(x)=x^{n} \sum_{p=0}^{n}(-n / x)^{p} / p$ !. J. D. Buckholtz points out that if $M=3.5911 \cdots$ is the number which satisfies $M \log M=M+1$, then for $|z| \leqq 1 / M,\left|z e^{1-z}\right| \leqq 1$ and $|z| \leqq 1$ and so by theorem 2 of [2], (see also [3]) $\sum_{p=0}^{n}(n z)^{p} / p ! \neq 0$. Thus the roots $z_{1}, \cdots, z_{n}$ of $Q_{n}$ must have modulus less than $M$, which completes the proof of Lemma 3.5.

We now use Lemma 3.5 to complete the proof of Theorem 3.2. Given $n$, choose $z_{1}, \cdots, z_{n}$ as in Lemma 3.5. Then for $|z|<1 / M$,

$$
\frac{1}{n} \sum_{i=1}^{n} g_{0}\left(z z_{i}\right)=b_{1} z+\sum_{m=n+1}^{\infty}\left[\frac{1}{n} \sum_{i=1}^{n} z_{i}^{m}\right] b_{m} z^{m} .
$$

The left side is in $\operatorname{co}\left(g_{0}(D)\right)$ and differs from $b_{1} z$ by a vector of norm less than or equal to

$$
\sum_{m=n+1}^{\infty}\left|b_{m}\right||z M|^{m}
$$

Since the power series expansion for $g(z)$ converges absolutely for 
$|z|<1$ [6, Theorem 3.11.4], the same is true of $g_{0}(z)$ and we get $\lim _{n \rightarrow \infty} \sum_{m=n+1}^{\infty}\left|b_{m}\right||M z|^{m}=0$, for all $z$ with $|z|<1 / M$. Hence $b_{1} z$ is in $\operatorname{cl}\left(\operatorname{co~} g_{0}(D)\right)$ for $|z|<1 / M$ and, as we have seen above, this completes the proof of Theorem 3.2.

4. The theorem in particular $B$-spaces. We have reduced the study of strong form of the maximum modulus theorem in any given space to the study of the complex extreme points of the unit sphere of that space.

In strictly convex spaces, such as $L_{p}(S, \Sigma, \mu), 1<p<\infty$, every point on the unit sphere is real extreme so the notions of real and complex extreme points coincide. They also coincide in sup norm spaces. For example, in $B C(S)$, the set of bounded continuous complex valued functions on an arbitrary topological space $S$, (a continuous) $f$ is real or complex extreme if and only if $|f(s)|=1$ for all $s$ in $S$. The same characterization holds in $L_{\infty}(S, \Sigma, \mu)$ with equality taken a.e. The strict convexity of the complex disc shows such $f$ are real extreme points. That other $f$ are not complex extreme is obvious in $L_{\infty}$. To see that they are not in $B C(S)$, suppose $|f|=1$ but $|f(s)|<1$ for some $s$. Then $g(s)=1-|f(s)|$ is not identically zero and $|f(s)+z g(s)| \leqq|f(s)|+1-|f(s)|=1$ for all $s$ in $S$ and all $z$ in the closed unit disc so $f$ is not complex extreme.

Though the notions of real and complex extreme points of ten coincide, allowing the easier Corollary 3.4 to settle the problem of the strong form of the maximum modulus theorem, the notions differ significantly in the case of $L_{1}(S, \Sigma, \mu)$, as we show below in Theorem 4.2 .

Lemma 4.1. Let $(S, \Sigma, \mu)$ be a measure space. For $f$ in $L_{1}(S, \Sigma, \mu)$, let $S(f)=\{\sin S: f(s) \neq 0\}$. For $f$ and $g$ in $L_{1}(S, \Sigma, \mu),|f+g|=|f|+|g|$ if and only if $f=h g$ a.e. on $S(f) \cap S(g)$ and $h(s)>0$ for $s \in S(f) \cap S(g)$.

Proof. Since $\int_{S}(|f|+|g|-|f+g|) d \mu=0$ we have $|f(s)+g(s)|$ $=|f(s)|+|g(s)|$ a.e. and an elementary calculation with complex numbers now shows that $f(s)=h(s) g(s)$ a.e. on $S(f) \cap S(g), h(s)>0$ : letting $f(s)=h(s) g(s),|1+h(s)||g(s)|=|g(s)+f(s)|=|g(s)|+|f(s)|$ $=(1+|h(s)|)|g(s)|$. Thus $|1+h(s)|=1+|h(s)|$ since $g(s) \neq 0$ and we see that $h(s)=|h(s)|>0$ for $s$ in $S(f) \cap S(g)$.

The sufficiency is clear.

THEOREM 4.2. Every point on the surface of the unit sphere of $L_{1}(S, \Sigma, \mu)$ is a complex extreme point.

Proof. Suppose that $f$ and $g$ are in $L_{1}(S, \Sigma, \mu)$ with $|f|=1$ and $|f+z g| \leqq 1$ for all $|z| \leqq 1$. As in the beginning of the proof of Theorem 
3.1, $|f+z g|=1$ for all $|z| \leqq 1$. Note further that $S(g) \subset S(f) \cup N$ with $\mu(N)=0$. To see this,

$$
\begin{aligned}
2=2 \int_{S(f)}|f| & \leqq \int_{S(f)}|f+g|+|f-g| \\
& \leqq \int_{S(f)}|f+g|+|f-g|+2 \int_{S-S(f)}|g| \\
& =\int_{S}|f+g|+|f-g|=2 .
\end{aligned}
$$

Thus $\int_{S-S(f)}|g|=0$.

-Write $f=(f+z g) / 2+(f-z g) / 2$ and note that $|f|=|f+z g| / 2$ $+|f-z g| / 2$, and applying Lemma 4.1 we can find for each $z$ a function $h_{z}$ so that we have $(f+z g) / 2=h_{z}(f-z g) / 2$ with $h_{z}(s)>0$, true a.e. on $S(f-z g) \cap S(f+z g)$. Then $z g=\left(h_{z}-1\right) f /\left(h_{z}+1\right)$ a.e. there. Let $\operatorname{sgn}(f(s))=\operatorname{cl}(f(s)) /|f(s)|$ if $f(s) \neq 0$ and $\operatorname{sgn}(f(s))=0$ if $f(s)=0$. We then have $z g \operatorname{sgn} f=\left(h_{z}-1\right)|f| /\left(h_{z}+1\right)$. The equation holds for all complex $z$ with $|z|<1$ and the right side is real. The equation for each $z$ is valid only for $s$ in $S(f-z g) \cap S(f+z g)$, but by taking $z$ sufficiently small it follows that $g$ sgn $f=0$ a.e. on $S(f)$, that is that $g=0$ a.e. on $S(f)$. But $g$ is zero a.e. on the complement of the support of $f$, and this completes the proof.

Suppose that $f$ is in $L_{1}(S, \Sigma, \mu)$ with norm one and $g$ is in $L_{1}(S, \Sigma, \mu)$ with $|f \pm g| \leqq 1$. Then $|f+\alpha g| \leqq 1$ for $-1 \leqq \alpha \leqq 1$ and as in the proof of Theorem 4.2, $g$ sgn $f$ is real, $g$ vanishes outside the support of $f$ and $\mid f \operatorname{sgn} f \pm g$ sgn $f \mid \leqq 1$. So we see that $f$ is a real extreme point in $L_{1}(S, \Sigma, \mu)$ if and only if $f \operatorname{sgn} f=|f(\cdot)|$ is a real extreme point in real $L_{1}(S, \Sigma, \mu)$. The extreme points in real $L_{1}(S, \Sigma, \mu)$ are characterized $\left[4\right.$, p. 81] as exactly those functions of the form $\pm C_{A} / \mu(A)$, where $A$ is an atom in $\Sigma$ and $C_{A}$ is the characteristic function of $A$.

In particular, the complex Banach space $L(0,1)$ has a unit sphere with no real extreme points but whose surface consists entirely of complex extreme points.

Our earlier remarks on $B C(S)$ and $L_{\infty}(S, \Sigma, \mu)$, in conjunction with Theorem 4.2, show us that the complex spaces $L_{\infty}(S, \Sigma, \mu)$ and $L_{1}\left(S^{\prime}, \Sigma^{\prime}, \mu^{\prime}\right)$ are never congruent (unless both are one-dimensional). If $B C(S)$ has a continuous function $f$ with $|f(s)|$ not constant, then we may conclude that complex $B C(S)$ and $L_{1}\left(S^{\prime}, \Sigma, \mu\right)$ are likewise never congruent (unless both are one-dimensional).

If $M$ is a subspace of $X$ then any extreme point of $S_{X}$ which is in $M$ is also an extreme point of $S_{M}$. Thus if the strong form of the 
maximum modulus theorem holds for $X$ it holds for all spaces congruent to subspaces of $X$. In the preceding paragraph, $L_{1}(S, \Sigma, \mu)$ can be replaced by any subspace thereof. In particular, the complex twodimensional Banach space $l_{\infty}^{2}$ is not congruent to any subspace of complex $L(0,1)$. In contrast Lindenstrauss has shown in [7] that every real two-dimensional Banach space is congruent to a subspace of real $L(0,1)$. If $X=X_{1} \oplus \cdots \oplus X_{n}$, where

$$
|x|=\left(\left|x_{1}\right|^{p}+\cdots+\left|x_{n}\right|^{p}\right)^{1 / p}, \quad 1 \leqq p<\infty,
$$

defines the norm for $x=\left(x_{1}, \cdots, x_{n}\right)$, note that a point is complex extreme for $S_{X_{i}}$ if and only if it is complex extreme when considered as a point of $S_{X}$. For suppose $x$ is extreme in, say, $S_{X_{1}}$. Then $\left|x+z\left(y_{1}, \cdots, y_{n}\right)\right| \leqq 1$ implies $\left|x+z y_{1}\right|^{p}+\left|z y_{2}\right|^{p}+\cdots+\left|z y_{n}\right|^{p} \leqq 1$ for $|z| \leqq 1$. But since $x$ is complex extreme in $S_{X_{1}}, y_{1}=0$, and $\left|x+z y_{1}\right|=1$, whence $y_{2}=\cdots=y_{n}=0$. Thus $x$ is complex extreme in $S_{X}$.

A rather different discussion of maximum modulus theorems appears in [1]. An easy example given there shows that the strong form of the maximum modulus theorem fails for $B(H)$, the set of bounded operators on a Hilbert space (of two or more dimensions).

\section{REFERENCES}

1. Arlen Brown and R. G. Douglas, On maximum theorems for analytic operator functions, Acta Sci. Math. 26 (1966), 325-327.

2. J. D. Buckholtz, A characterization of the exponential series, Amer. Math. Monthly 73 (1966), 121-123.

3. - Sums of powers of complex numbers, Notices Amer. Math. Soc. 13 (1966), 372.

4. M. M. Day, Normed linear spaces, Springer-Verlag, Berlin, 1958.

5. N. Dunford and J. Schwartz, Linear operators, Vol. I, Interscience, New York, 1958.

6. E. Hille and R. S. Phillips, Functional analysis and semi-groups, rev. ed., Colloq. Publ., Vol. 31, Amer. Math. Soc., Providence, R. I., 1957.

7. J. Lindenstrauss, On the extension of operators with a finite-dimensional range, Illinois J. Math. 8 (1964), 488-499.

8. J. V. Uspensky, Theory of equations, McGraw-Hill, New York, 1948.

University of California, Irvine and

UNIVERSITY OF MARYLAND 